\section{Biosorption at the HES-SO Valais-Wallis Sion Looking for Partners to Implement Innovative Technologies}

Elsbeth Heinzelmann, Science and technology journalist

\section{Based on information from Professor Simon Crelier, HES-SO Valias-Wallis}

\begin{abstract}
Water pollution contributes to a number of health problems and disorders in humans, and dramatically affects wild animals and the environment as a whole. Biosorption is a sound and sustainable way to remove pollutants like metals, dyes, phenols, pesticides or drug metabolites from liquid effluents. But despite active research worldwide, technical limitations still impede its widespread use. At HES-SO ValaisWallis in Sion, Prof. Simon Crelier is working on the development of efficient, economically viable and scalable biosorption processes.
\end{abstract}

\section{Keywords: Adsorption isotherm - Adsorption kinetics . Biosorbent · Effluent treatment · Micropollutants · Scale-up}

Biosorption is a naturally-occurring physicochemical process in which biomass passively binds environmental contaminants. By adsorption it removes undesirable soluble substances organic or inorganic - from solutions using inexpensive biological materials. The application of biosorption as a process primarily requires expertise in chemical engineering. This approach has been tested in a variety of situations as it is simple and efficient thanks to the availability of biomass and waste bio-products. However, to date there has been little or no exploitation of this technique in an industrial context. Professor Simon Crelier, a researcher in the Life Technologies Department at HES-SO Valais-Wallis in Sion is working to change this situation.

\section{What Enemy Are We Fighting against?}

Although contamination is occasionally of natural origin (e.g. arsenic-poisoned groundwater in India, Bangladesh or Argentina), micropollutants are chemical substances usually derived from human activities, such as pesticides, detergents, heavy metals, biocides, drugs and drug metabolites, cosmetics or nanoparticles. These substances, although present at low concentrations, gradually accumulate in the environment, where their negative impact on ecosystems has been demonstrated. They are thus under intense scrutiny, and a lot is being done in terms of monitoring of their concentrations in air, water and soils. On the other hand, much less is proposed when it comes to the removal of micropollutants.

There are many chemical or physicochemical approaches that would facilitate the reduction or destruction of these contaminants. Generally-speaking, the closer one is to the source of contamination, the more concentrated the pollutant and the easier it will be to solve the problem. Things get more complicated when you go downstream, and once you reach the wastewater treatment plant you have to deal with complex mixtures of highly diluted substances. Fortunately, it is at low concentrations that adsorption works best, leading to a significant concentration of the adsorbate on the solid phase, thus facilitating proper disposal.

\section{What is an 'Efficient Biosorbent'?}

Biosorbents can be obtained from a wide variety of materials, by-products or waste. Candidate adsorbents have to meet several requirements to be eligible: they have to be cheap, available in large quantities, sustainable and recyclable, highly efficient with respect to capacity and mass transfer and, last but not least, scalable. Activated carbons, algae, sawdust, rocks, egg shells and others are worthy of consideration. While the scientific literature features an incredible diversity of examples, these seldom address the following question: are these biosorbents able to compete with synthetic, commercial adsorbents, which usually consist of regularly shaped, mechanically resistant beads with high specificity and capacity?

The performance of a given matrix can usually be improved by chemically altering its surface to make it more selective or increase its capacity. Reusability is also a key element, i.e. you must be able to release the adsorbate and regenerate the adsorbent surface before starting a new cycle. However the more processing and engineering goes into the preparation, surface chemistry and formulation of the biosorbent, the more expensive it will become. An awareness of process economics will help the engineer decide where to stop.

\section{How Can We Characterize Adsorbents?}

"Biosorbents are characterized exactly in the same way as chromatography resins" explains Simon Crelier. "And the same type of experiment is performed to this effect". He believes that we need to address the following questions at the start of any biosorbent development:

What can I adsorb with this material? Depending on the surface nature and chemistry, the material will be able to trap heavy metal ions by electrostatic interactions or ion exchange. Others will feature interesting properties for reducing hydrophobic, organic molecules. This will also define the selectivity of the adsorbent in relation to specific molecules.

How much can I adsorb with this material? What is important here is the adsorbent capacity at equilibrium. To determine this capacity, given amounts of fresh adsorbent are contacted with a certain volume of adsorbate solution of known initial concentration and left under agitation until equilibrium is reached.

A line plotting the adsorbed concentration at equilibrium as a function of the residual adsorbate concentration in the liquid phase is called an isotherm. Despite a large number of models, three simple equations are usually sufficient to describe most liquid/ solid adsorptions: linear, Langmuir or Freundlich isotherms.

How fast is the adsorption process? The kinetics of the adsorption process is usually described using models very similar to chemical reaction kinetics - although no reaction is taking place. These measurements consist in monitoring the course over 
time of the adsorbate concentration in the liquid or on the surface of the adsorbent.

Here too, the kinetics may be described by a range of models, which can either be descriptive/empirical or based on mass transfer considerations.

Measuring isotherms at different temperatures allows the process to be characterized thermodynamically and gives access to such parameters as $\Delta \mathrm{G}^{\circ}, \Delta \mathrm{H}$ and $\Delta \mathrm{S}$ (Gibbs free energy, enthalpy and entropy of adsorption, respectively). By determining the adsorption rate constant at different temperatures and using the Arrhenius equation, one can also obtain the energy of activation of the adsorption process.

The scientist insists: "This information is extremely important for the design and calculation of any adsorption device or plant. Without it you lay yourself open to unexpected problems. Since the experimental set-up and the analytics are usually pretty standard (although we are dealing here with trace concentrations), these trials really are worth the time spent on them".

But this is still not the end of the story, as Simon Crelier points out: "You could have the best adsorbent in terms of capacity and selectivity, but you also need to condition it in such a way that the particles will possess sufficient rigidity and mechanical resistance while having good mass transfer characteristics."

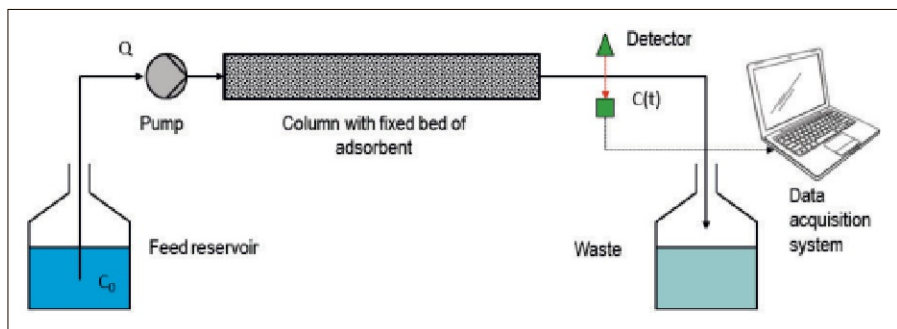

Typical experimental setup for the measurement of fixed bed adsorption. The solution of adsorbate is pumped continuously through a fixed bed of adsorbent. The concentration in the effluent is monitored as a function of time, yielding the corresponding breakthrough curve. (Figure: Simon Crelier HES-SO Sion)

\section{Focus on an Interesting Approach}

A frequently used industrial application in wastewater treatment is fixed bed adsorption. Although batch dispersion is

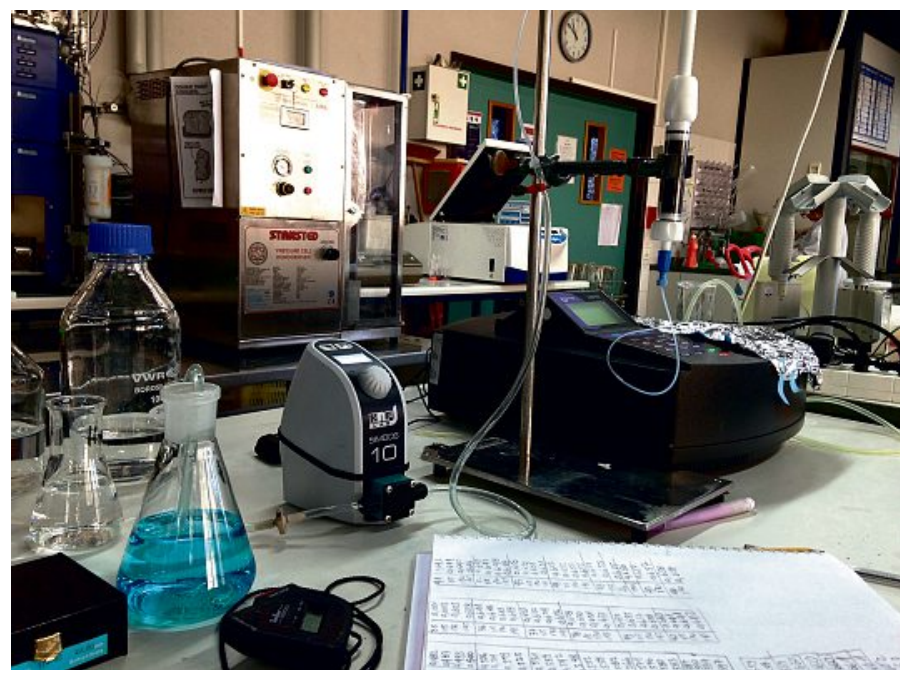

A flask of contaminated effluent, a pump, a column with a fixed bed of adsorbent and a detector: a simple laboratory set-up can provide very useful information on adsorbent capacity and mass transfer. (Simon Crelier HES-SO Sion) by far the most efficient configuration in terms of mass transfer and kinetics, it requires large tank volumes and footprints on an industrial scale. Fixed bed operation with three columns working in sequence - pre-loading, loading and elution/regeneration makes much better use of the available space.

In this configuration the effluent is continuously pumped through the column and the loading of the adsorbent gradually increases until equilibrium is reached between the entering and the adsorbed concentration. Column switching is usually done earlier in order to avoid excessive release of the solute. A breakthrough curve results from measuring the adsorbate concentration in the exiting effluent as a function of time, processed volume or loaded adsorbate quantity. This $\mathrm{S}$-shaped curve provides a useful insight into the mass transfer conditions in the fixed bed. The curve can be described by different models, most of which are empirical. "As a matter of fact the accurate modelling of such curves is made difficult by the fact that concentrations vary as a function of both time and location in the column", explains Simon Crelier. "But if you want to understand how your fixed bed is performing or if you wish to predict it from the data obtained in batch experiments, there is no other way than to incorporate the influence of flow conditions, particle size, mass transfer and diffusion coefficients in the differential mass balances."

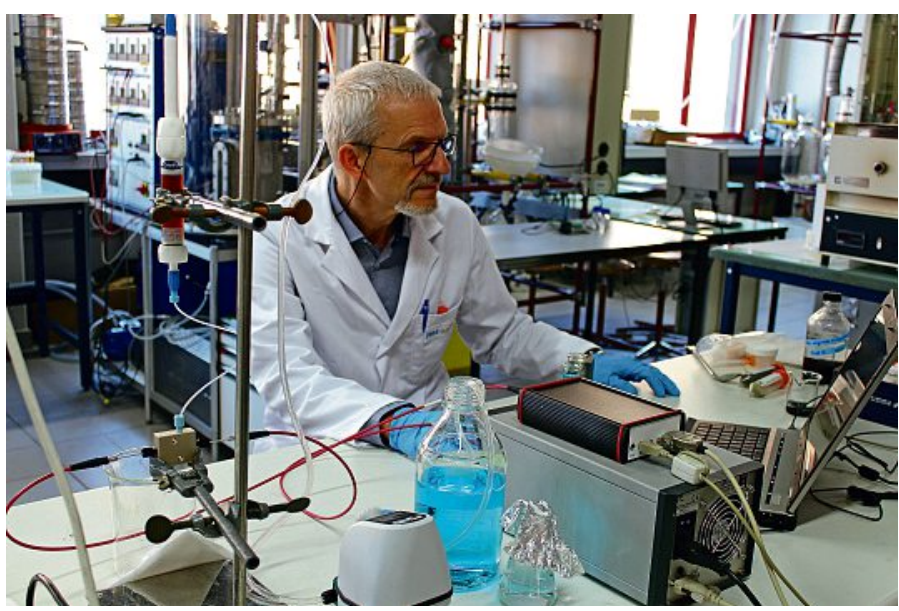

Experimental set-up for the measurement of breakthrough curves. Prof. Simon Crelier is configuring the fiber optics spectrophotometer used for concentration measurements in the effluent. The detection cell can be seen after the adsorbent fixed bed in the bottom left of the picture. (Photo Elsbeth Heinzelmann)

\section{Moving Away from Lab-scale Biosorption}

Over the past few years, Crelier's group have worked on different systems, gaining experience and expertise in the characterization and implementation of biosorbents. By way of example, they removed copper ions from an aqueous solution using chitosan microbeads or powdered brown algae. In another project they treated synthetic effluents containing carbamazepine or removed chromium ions $(\mathrm{Cr}(\mathrm{VI}))$ using Laminaria digitata algae, or phenol using a 'home-made' activated carbon derived from apricot stones. They also managed to extract lipophilic molecules in alginate beads loaded with encapsulated oil vesicles. "We found this approach much easier than producing microcapsules with a liquid core and a thin alginate membrane", points out Simon Crelier. "Alginate beads with oil contents as high as $70 \% \mathrm{v} / \mathrm{v}$ were obtained by emulsifying the oil in an alginate solution and solidifying the emulsion in a calcium chloride solution." 


\section{The Trick with Magnetic Beads}

A highly promising technology for the removal and recovery of contaminants from liquid effluents involves bio-functional magnetic beads and is the subject of experimentation by Simon Crelier's group. "Since a stirred tank is the most efficient configuration for performing adsorption in terms of mass transfer, we thought about possible ways to separate the adsorbent particles from the treated liquid prior to adsorbate elution and bead regeneration: a filter element could be used, but this is prone to clogging if the effluent contains solid particles. Our approach consists in using magnetic adsorbent beads. These were easily produced by encapsulating maghemite inside the alginate matrix. At the end of the adsorption cycle, the beads are easily collected by applying a magnet to the external wall of the stirred tank. The decontaminated liquid can then be drained, the adsorbate eluted and the adsorbent prepared for a new cycle. Together with Prof. Careño-Morelli and Prof. Chevailler, two colleagues from the Institute of Systems Engineering, we are developing a smallscale demonstrator employing this approach". a solution", says Simon Crelier, whose laboratory is integrated in the Biotechnology Unit at the HES-SO Valais-Wallis. Not surprisingly, the Unit's research activities increasingly involve environment-related projects. "Under laboratory conditions, biosorption efficiently eradicates heavy metals from industrial wastewater or soil contaminants, and can even be used to purify precious metals like gold or silver", adds the researcher, who has 25 years of experience in research. "But each new method, even if highly innovative, has to be cost-efficient and guarantee the repeated absorption of adequate quantities. What remains has to be recycled - as in the case of precious metals - or properly disposed of."

One reason why biosorption is so popular is that lab-scale characterization is very easy to perform and does not require a lot of fancy equipment. Things get more complicated when you need to scale things up to real-life conditions, and this might explain in part why so few companies out there are offering equipment or services in the biosorption field. One striking exception is BV Sorbex, a Canadian company created by Prof. Bohumil Volesky, a pioneer of biosorption. "But the time has come to put
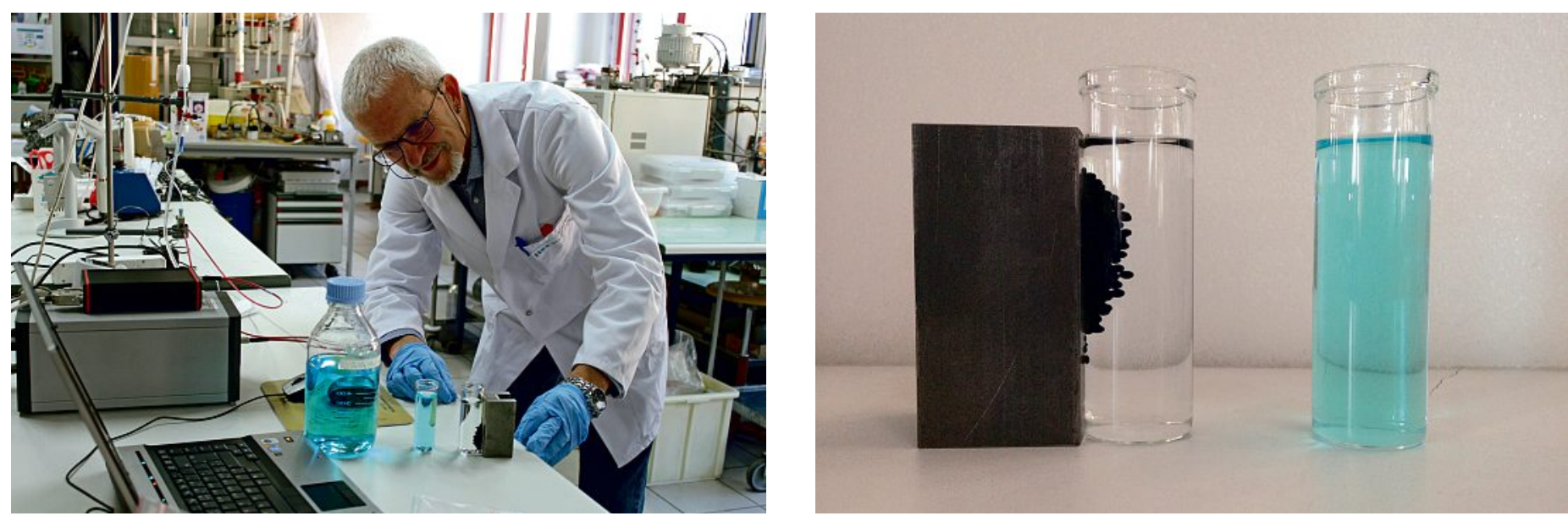

Separating magnetic adsorbent beads from treated effluent containing copper ions. The original blue-colored copper sulphate solution can be seen in the left-hand tube. After complete removal of the metal ions, the beads can be sequestered using a simple magnet, the decontaminated liquid released, the copper released in a more concentrated form allowing proper reuse and the adsorbent regenerated. (Photos Elsbeth Heinzelmann)

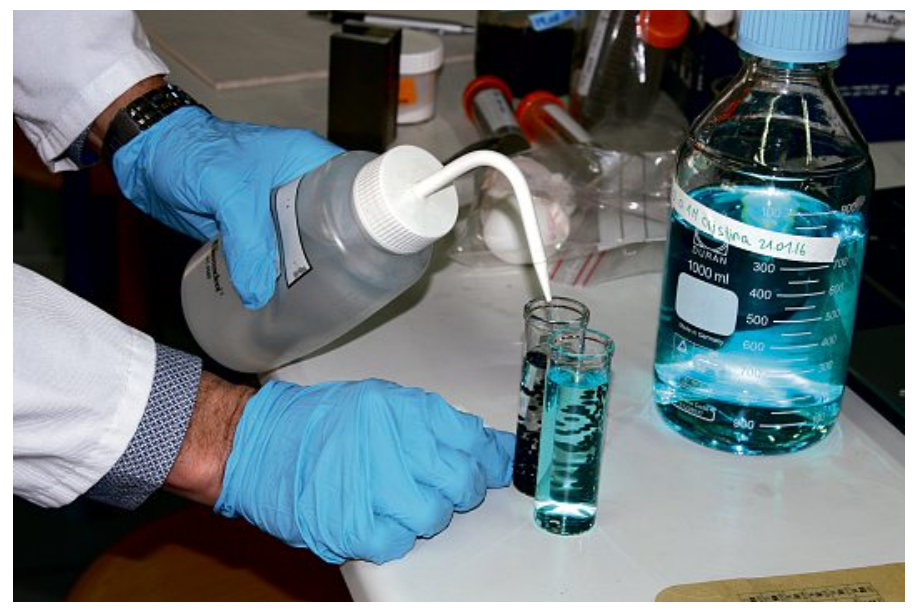

Suspending composite chitosan/maghemite magnetic adsorbent particles in a copper sulphate solution. (Photo Elsbeth Heinzelmann)

\section{The Key to Success}

Around the globe extensive research is being carried out on biosorption methods utilizing the surface properties of inert biomass (whether bacteria, orange peels, activated carbon or minerals) for environmental clean-up. "Biosorption is the method of choice for removing metal ions or organic compounds from

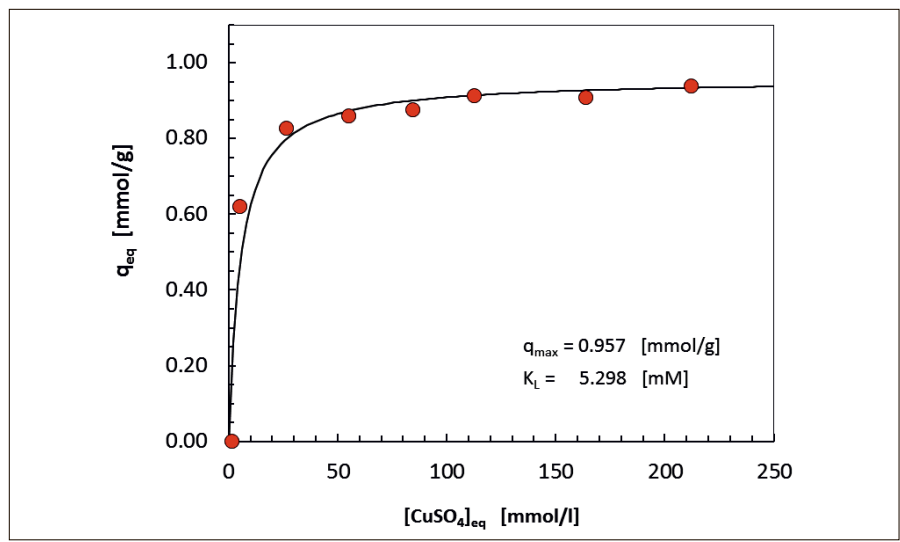

Adsorption isotherm of copper ions on a commercial resin at room temperature, indicating the adsorbed concentration at equilibrium with the corresponding residual concentrations of adsorbate in the liquid phase. In this example it is the Langmuir isotherm that best describes the experimental data. (Graph Simon Crelier HES-SO Sion)

our expertise to the test", states Simon Crelier, who is actively seeking partnerships to further develop and bring biosorption to industrial maturity. "Please do not hesitate to contact us!"

www.itv.hevs.ch

simon.crelier@hevs.ch

Received: March 31, 2016 